Meta

Journal des traducteurs

Translators' Journal

\title{
Définition d'une démarche terminologique en fonction des besoins à satisfaire
}

\section{Robert Dubuc}

Volume 18, numéro 1-2, mars 1973

Actes du deuxième colloque international de linguistique et de traduction. Montréal, 4-7 octobre 1972

URI : https://id.erudit.org/iderudit/002626ar

DOI : https://doi.org/10.7202/002626ar

Aller au sommaire du numéro

Éditeur(s)

Les Presses de l'Université de Montréal

ISSN

0026-0452 (imprimé)

1492-1421 (numérique)

Découvrir la revue

Citer cet article

Dubuc, R. (1973). Définition d'une démarche terminologique en fonction des besoins à satisfaire. Meta, 18(1-2), 269-276. https://doi.org/10.7202/002626ar d'utilisation que vous pouvez consulter en ligne. 


\section{Définition d'une démarche terminologique en fonction des besoins à satisfaire}

\section{ORIENTATION GÉNÉRALE}

Quand il s'est agi de jeter les bases d'une banque de terminologie à l'Université de Montréal, avec la caution de l'Office de la langue française du Québec et du Bureau des traductions du Secrétariat d'État fédéral, trois ordres de critères sont entrés en ligne de compte : les principes de recherche terminologique admis par les organismes de terminologie, les besoins du milieu à desservir et les utilisations subsidiaires à des fins de recherche linguistique et lexicographique.

1.1. Principes de recherche terminologique. - Lors du Congrès de terminologie tenu à Genève en décembre 1969 , les participants, représentant les principaux organismes de terminologie internationaux et les universités intéressés à la recherche terminologique et à la traduction, se sont entendus sur trois principes fondamentaux susceptibles d'assurer à la recherche terminologique un seuil de validité acceptable.

1.1.1. Ces principes sont les suivants: a) l'information terminologique doit toujours être citée dans son contexte; $b$ ) elle doit porter la mention de source et la date ;c) elle doit être tirée de textes originaux dignes de foi.

1.1.2. Pourquoi le contexte? Un mot ne vit qu'en situation. Hors contexte, le mot est mort. En ce sens, on a raison de dire que les dictionnaires sont les sarcophages des mots de la langue. On sait aussi que les mots d'une langue sont polysémiques presque par essence, la dérivation sémantique étant l'une des principales sources de la création lexicale. D'après le Grand Larousse encyclopédique, le seul mot MAIN comporte 35 sens techniques différents, allant de l'automobile à la zoologie, en passant par l'équitation, la marine et la pêche. L'utilisation du contexte permet d'étudier les termes en situation dans chaque langue de travail. Il est ainsi possible non seulement de trouver les équivalents de terme à terme, mais encore d'accéder aux formes d'expression spontanée propres à chaque langue de travail. On ne touche plus uniquement aux termes strictement techniques, mais à tout le vocabulaire général d'application scientifique qu'il est nécessaire de connaître si l'on veut s'exprimer de façon idiomatique. C'est l'étude des termes en situation qui nous apprend que to convert sound waves into electric current se dit généralement en français « transformer les ondes sonores en courant électrique $\gg$. TRANSFORMER et non convertir. Cette démarche terminologique 
bilingue ne subordonne pas une langue à l'autre, mais met plutôt les langues en parallèle.

1.1.3. La mention de source et de date. - La mention de source répond à des exigences de validité et de commodité. En effet la qualité de l'information donnée dépend souvent de la valeur de la source d'où elle est tirée. Comme source d'un terme de biologie, l'article à sensation de Paris-Match a certainement moins de valeur qu'une monographie de Jean Rostand. La mention de source peut se révéler utile pour permettre au consultant l'accès à des développements supplémentaires. Dans ce cas, il faut que la mention de source soit liée à une référence bibliographique complète, établie selon les normes généralement reçues.

La mention de date s'impose en particulier pour la terminologie scientifique et technique. À une époque de découvertes accélérées, un écart de 10 ou 15 ans peut être lourd de conséquence. Dans les techniques de pointe comme l'informatique, une marge de deux ou trois ans peut même être significative.

1.1.4. L'insistance sur la recherche fondée sur des textes originaux a été, à ce qu'il semble, occasion de scandale. Cette exigence serait excessive selon certains. C'est sous-estimer les risques de contamination en traduction, surtout si l'on tient compte des contraintes de temps imposées le plus souvent aux traducteurs. Il faut briser quelque part le cercle vicieux de la traduction qui mène à la création d'une langue de traduction parallèle à la langue vivante et calquée sur la langue de départ. Le traducteur qui rend opening of a parachute par «ouverture d'un parachute » ne pêche pas contre la langue, mais il ignore que, dans l'usage français, l'expression «déploiement d'un parachute » est tout aussi courante ; de même celui qui rend automatiquement test tube par «tube à essai» peut ne pas être dans l'erreur, mais que devient alors le mot courant «éprouvette»?

Le piège des mots-sosies, les contraintes de temps, l'absence de connaissances $\mathrm{du}$ sujet traduit constituent autant de raisons qui justifient la prévention contre la recherche terminologique à base de textes traduits l'un de l'autre. Il n'est bien sûr jamais de règles si absolues qu'elles n'impliquent aucune exception. Dans les domaines de pointes, il faut parfois se rabattre sur des textes traduits, en l'absence de toute autre documentation. Il faut alors être particulièrement exigeant sur la qualité de la traduction.

On dit aussi «textes dignes de foi », puisqu'il va sans dire que la qualité de l'information est tributaire de la valeur de la source d'où elle est tirée. Les textes qui jouissent d'une certaine autorité dans le domaine étudié fournissent une matière de choix. Les manuels, en particulier, constituent de ce fait une excellente source de renseignements terminologiques.

1.1.5. Le premier principe appelle comme corollaire l'assimilation de la définition au contexte. Comme nous travaillons dans une optique proprement terminologique et non lexicographique, la valeur significative et définitoire du contexte prend une importance particulière. Plus le contexte s'apparente à une définition, mieux il s'adapte à nos fins.

1.2. Besoins du milieu. - La conception d'un projet de l'envergure d'une banque de terminologie ne pouvait être un simple travail de cabinet, conçu en serre 
chaude, sans tenir compte des besoins du milieu à desservir, de la façon dont les problèmes terminologiques s'y posent et des essais de réponse tentés jusqu'à ce jour.

1.2.1. Dans le contexte canadien, les problèmes de terminologie se posent, le plus souvent, en termes de traduction. Ce n'est pas par hasard que les traducteurs ont toujours été présents lorsqu'il a été question de banque de terminologie. Le colloque de Stanley House, où pour la première fois il a été question d'une banque de données terminologiques, avait été organisé par la Société des traducteurs et interprètes du Canada et c'est à un colloque sur la traduction, organisé en 1968 par l'Office de la langue française, que M. André Clas, alors directeur du Département de linguistique de l'Université de Montréal, a relancé l'idée qui devait permettre à ce projet de prendre la forme qu'on lui connaît aujourd'hui. Cette prépondérance de la traduction s'explique par notre situation géographique et économique, qui fait également que les problèmes se posent beaucoup plus de l'anglais au français qu'en sens inverse.

Voisins des Américains, nous recevons déjà étiquetées en anglais les innovations scientifiques et techniques qu'ils mettent au point. Les problèmes de terminologie scientifique et technique se posent rarement chez nous dans l'optique d'une création spontanée : tout passe plutôt par le canal de la traduction. Les autoneiges québécoises se sont d'abord appelées snowmobiles. Sur le plan psychologique, cette situation gêne sensiblement l'implantation de la terminologie française. L'acquisition d'une connaissance est toujours étroitement liée à une terminologie. Une fois cette association faite, il est très difficile d'en créer artificiellement une nouvelle. Plus la terminologie française tarde à être connue, moins elle a de chance de s'enraciner. Le problème de la diffusion prend de ce fait une importance primordiale.

1.2.2. En plus d'être bilingue, l'information terminologique doit donner aux aspects pratiques et concrets de la technique préséance sur les aspects théoriques. Pour un terme de technologie qu'on nous demande, il faut pouvoir en fournir dix sur les parties d'une fermeture-éclair, les différents types de bouchons dans l'ernballage ou les pièces d'une machine à fileter.

1.2.3. En raison de cette orientation, se pose de façon décisive le problème de la masse de l'information à accumuler. Quand on sait que pour le seul domaine de la quincaillerie, il y a plus de 32000 pièces distinctes à nommer, il devient évident que, pour satisfaire aux besoins du public et de sa clientèle, une banque de terminologie doit viser à un «capital de roulement» de l'ordre de 300000 fiches.

1.2.4. De plus, si la banque veut mieux répondre aux besoins de ses usagers que les dictionnaires de type classique, il lui faut pouvoir mieux qu'eux se tenir au diapason de l'usage, intégrer les mots nouveaux, annuler les mots désuets, corriger l'information fautive. Tous ces remaniements doivent s'opérer sur une base quasi quotidienne. Toute structure qui complique les opérations de correction doit donc être écartée. C'est ce qui explique que nous ayons éliminé les structures analogiques ou associatives de notre fichier. Essentielles dans une banque monolingue, ces structures prennent beaucoup moins d'importance dans une banque 
bilingue où la langue de départ - quelle qu'elle soit - fournit les indices de repérage de l'information. Chaque fiche doit donc être dans la mesure du possible autonome et complète.

1.2.5. Il ne suffit pas d'emmagasiner et de tenir à jour l'information, il faut encore donner à ceux qui en ont besoin un accès rapide au capital stocké. Ceux qui ont à un titre ou l'autre cuvré en traduction savent que les problèmes terminologiques s'y posent toujours avec une certaine urgence. Le délai de 48 heures apparaît comme un seuil au-delà duquel on ne saurait guère aller si l'on ne veut pas gêner sensiblement l'efficacité d'un système de consultation terminologique.

1.3. Les réponses du milieu. - Les usagers de terminologie n'ont pas attendu la création d'une banque de termes pour chercher à résoudre leurs problèmes. Très tôt, la fiche terminologique s'est imposée comme un instrument souple pour résoudre des problèmes terminologiques bien délimités. La majorité des fichiers actuels utilise la fiche 3 sur 5 po $(75 \times 125 \mathrm{~cm})$ comme support (Radio-Canada, Secrétariat d'État, Chemins de fer nationaux). On utilise aussi comme supports les vocabulaires (miméographiés ou imprimés à l'offset) sur format $8^{1 / 2}$ sur 11 . Ces vocabulaires prennent le plus souvent la forme de lexiques bilingues alphabétiques, mais parfois ils sont présentés sous une forme raisonnée à partir de centres d'intérêt.

Tous les travaux effectués à ce jour, au Canada, l'ont été selon des méthodes diverses. Ils sont, il faut bien le reconnaitre, de valeur souvent aléatoire, faute d'un corps de doctrine et d'une méthodologie généralement acceptée.

Ces travaux se bornent le plus souvent à un simple appariement de termes, sans indication de source, de domaine ni de contexte et sont par le fait même d'une utilité restreinte, en dépit des efforts qu'on a pu mettre à les établir.

Il faut faire exception des travaux d'orientation normative faits par l'Office de la langue française du Québec, dont les Cahiers s'emploient à définir les notions étudiées et à justifier les équivalents proposés et ceux du Comité de linguistique de Radio-Canada, qui suivent sensiblement la même méthode.

\section{LA FICHE TERMINOLOGIQUE DE LA BANQUE DE TERMINOLOGIE}

2.1. C'est à la lumière de ces principes et en tenant compte à la fois des besoins et des usages du milieu que la Banque de terminologie a mis au point sa fiche terminologique.

Il s'agit d'une fiche 3 sur 5 po à 17 champs d'information, essentiellement bilingue et réversible. Elle permet pour chaque langue de noter les vedettes, la source de l'information, l'année de publication, les références exactes, les marques d'usage, la pondération et les notations grammaticales qui ont une portée terminologique. La fiche porte en outre l'indication des domaines d'application, l'indicatif de l'auteur de fiche et un indice de pondération global portant sur la fiche entière.

2.1.1. Dans le champ des vedettes, on peut inscrire, jusqu'à concurrence des 58 caractères, l'expression au complet, plus un ou deux synonymes (attestés par le 
contexte). On peut aussi avoir accès à un élément simple de la vedette en soulignant le dernier caractère du mot à récupérer. Une inscription annexe peut donner accès à un élément complexe de la vedette, par exemple, la fiche wastewater treatment plant peut être accessible, si l'on fait l'inscription annexe, par l'élément complexe waste-water treatment. En outre, on peut mettre en vedette, en plus de la forme articulée, la forme abrégée d'une expression, lorsqu'il existe une abréviation consacrée par l'usage.

2.1.2. La source en code renvoie à la référence bibliographique complète. La combinaison du paramètre source avec un code de domaine permet de dresser automatiquement des bibliographies raisonnées.

2.1.3. Les marques d'usage circonscrivent le champ d'utilisation de la vedette en permettant d'en identifier la provenance géographique ou d'en déterminer l'aire sociale d'application, la fréquence, l'ancienneté ou les nuances de sens ${ }^{1}$ (caractère spécifique, générique, dépréciatif, etc.), soit enfin d'en préciser la portée didactique (distinguer le terme courant du terme savant, le terme d'argot de métier du terme technique officiel, la marque de commerce du terme commun, etc.).

Le terme motoneige doit être identifié comme canadianisme, le terme gramophone comme britannique, l'abréviation familière mike doit pouvoir être identifiée comme telle ; l'appariement dynamic microphone et MICROPHONE ÉLECTRODYNAMIQUE ne peut se justifier que si le premier est identifié comme terme spécifique et le second comme générique.

2.1.4. La pondération évalue, à titre indicatif, le terme proposé par la fiche, selon l'échelle suivante : 0 à proscrire, 1 douteux, 2 acceptable et 3 normalisé. L'expression « avantages marginaux» pour rendre fringe benefits serait pondérée à 0 , l'emprunt «marketing » en français, à 1 parce que son emploi apparaît moins fonctionnel que celui du terme français commercialisation, qui serait coté à 2 . Enfin les termes MATÉRIEL - hardware et PROGRAMMERIE - software seraient pondérés à 3 puisque ces expressions ont été ratifiées par l'Association française de normalisation dont nous acceptons les décisions. La Banque ne fait aucun travail de normalisation, elle s'en remet plutôt à cette fin aux décisions des organismes officiels dont, entre autres, l'Office de la langue française du Québec, l'A.F.N.O.R., l'International Standard Organization (I.S.O.).

2.1.5. Les marques grammaticales ne sont retenues que s'il y a une motivation terminologique à leur inscription : genre flottant, catégories grammaticales imprécises, etc. L'expression MANCHE ̀̀ AIR - wind sleeve devra porter la mention F pour féminin, puisque au Canada le genre en est flottant. Le terme oPÉRANDE pour rendre l'anglais operand devra porter les mentions $\mathbf{N}$ (nom) $\mathbf{M}$ (masculin) parce que sa forme ne peut permettre de déterminer avec certitude ni sa catégorie grammaticale ni son genre.

1. On touche ici aux critères de la lexicologie différentielle que $\mathbf{M}$. Jean Darbelnet expose ailleurs dans le présent numéro. La démarche de la Banque, à ce stade de son exploitation, n'a qu'une portée indicative. Elle ne peut prétendre offrir autre chose à l'usager que des points de repère qu'on peut trouver vagues et arbitraires mais qui sont néanmoins indispensables dans le contexte canadien. 
2.1.6. Les domaines d'application sont déterminés en code à l'aide de la classification générale établie par la Banque, selon une structure logique à trois niveaux, utilisant l'alphabet comme indicatif. Cette classification offre une capacité virtuelle de 17500 domaines. C'est sur la recommandation des responsables des banques européennes ${ }^{2}$ que la Banque de l'Université de Montréal a décidé de mettre au point son propre système de classement, plutôt que d'adapter les classifications déjà existantes (Dewey, décimale, Library of Congress) dont l'orientation nettement bibliothéconomique en rend le maniement onéreux en terminologie. Ces classifications interdisent à toute fin pratique l'indication de plus d'un domaine par expression ; la nôtre au contraire facilite l'attribution multiple des domaines avec tous les avantages qu'une telle souplesse entraîne.

Dans chaque code de domaine, la première lettre correspond à la classe, c'est-à-dire à la discipline ou au domaine général d'application. La lettre $\mathrm{C}$ en première position représente le domaine de la chimie. La seconde lettre correspond à la division, c'est-à-dire à la sphère d'activité visée, au sein de la discipline. Pour la chimie, la lettre $\mathrm{A}$ en deuxième position renvoie à la chimie générale, la lettre $\mathrm{B}$ à la chimie analytique, la lettre $\mathrm{C}$ à la chimie minérale..., la lettre $\mathrm{F}$ aux matières plastiques. La troisième lettre désigne la section, c'est-à-dire le pôle immédiat de regroupement de l'information.

2.1.7. Comme la Banque de terminologie de l'Université de Montréal a été conçue pour être une immense «coopérative» d'information terminologique, il était nécessaire de pouvoir identifier la provenance des fiches de façon à respecter la propriété intellectuelle du fournisseur. Un code a donc été prévu à cette fin, qui nous permet de dresser automatiquement la liste de toutes les fiches fournies par un même rédacteur. Jusqu'ici, ce sont surtout les étudiants de l'École de traduction qui ont contribué à la Banque et leur apport nous a été extrêmement précieux.

2.1.8. Enfin, l'indice de pondération globale n'évalue plus seulement chaque vedette de la fiche, mais aussi leur correspondance d'une langue à l'autre. C'est toutefois la même échelle qui s'applique. Par exemple, si l'on rend l'expression voice coil simplement par bobine, chaque vedette en soi est excellente et peut recevoir la pondération 2. Mais l'équivalence est trop partielle pour qu'on ne mette pas l'usager en garde par une pondération inférieure à 2 .

\section{EXPLOITATION DE LA BANQUE}

3.1. Ainsi conçue, la fiche respecte essentiellement les principes du Congrès de Genève; elle suit aussi, en l'améliorant, la pratique courante des centres de terminologie quant au format et au contenu de la fiche. Ce qui devrait faciliter

2. La Banque de terminologie tient à souligner la précieuse collaboration qu'elle a eue de la part des représentants d'organismes européens, en particulier de MM. A. Bachrach et $\mathbf{J}$. Goetschalckx, du Bureau de traduction de la Communauté européenne à Luxembourg, et de M. F. Krollmann du Service de traduction de l'Allemagne fédérale. En nous faisant part de leur expérience, ils ont grandement éclairé notre orientation. A ces noms, il convient d'ajouter celui du professeur Jean-Paul Vinay, de l'Université de Victoria (C.B.), dont les travaux de recherche sur l'automatisation des dictionnaires nous ont été utiles à tous les stades d'élaboration de notre projet. 
l'intégration de l'information, son utilisation et la collaboration entre les divers organismes intéressés.

3.2. Pour la diffusion de l'information, la Banque envisage trois modes de consultation.

3.2.1. La consultation en accès direct par terminal assurera la solution immédiate de problèmes terminologiques urgents. Ce mode de consultation sera d'une grande utilité pour tous les organismes à grand débit de traduction. Les expériences d'automatisation de fichier confirment non seulement l'accélération du rendement des traducteurs mais aussi l'amélioration de la rigueur terminologique.

3.2.2. La consultation peut aussi se faire en différé lorsqu'il s'agit par exemple d'obtenir une masse considérable de renseignements : les termes propres à un secteur donné, la liste de termes par source, etc., ou encore les listes provenant de la combinaison de deux ou plusieurs paramètres terminologiques : domaine et source, domaine et marques d'usage, domaine et pondération, etc.

3.2.3. Ces deux services de base sont complétés d'un service de consultations téléphoniques ou par correspondance afin de répondre aux besoins d'une clientèle qui ne pourrait investir les sommes requises pour l'abonnement direct. Tout comme pour le traitement en différé, le délai de réponse ne devrait pas excéder, en règle générale, 48 heures.

3.3. Le fichier de la Banque permet encore des utilisations subsidiaires aux fins de la recherche terminologique et lexicographique. En effet, en regroupant divers paramètres terminologiques, on peut, par exemple, obtenir par domaine la liste des néologismes, des américanismes ou des canadianismes. Il devient ainsi possible de fournir aux chercheurs un corpus de recherche quasi instantané. On peut encore faire établir la terminologie propre à un auteur, faire relever, par domaine ou par source, les termes selon leur pondération pour faciliter le travail de normalisation. Enfin, les contextes et les définitions recueillis peuvent constituer une matière brute très intéressante pour le travail de lexicographie spécialisée. À ce titre, la Banque justifie son rattachement à une institution universitaire.

3.4. Elle le justifie encore par sa contribution à la formation terminologique des futurs traducteurs. Grâce à la présence de la Banque à l'Université, les étudiants en traduction ont l'occasion de se rompre de façon méthodique à la recherche terminologique, d'acquérir une méthode de travail et de se sensibiliser à la qualité de l'information terminologique en respectant les normes de validité universellement reconnues.

3.5. Notons enfin que, grâce aux programmes mis au point par le Centre de calcul de l'Université de Montréal, notre système dresse automatiquement le bilan de son activité, et comptabilise, par terme, le nombre de consultations, fait le relevé des demandes non satisfaites, de façon à renseigner les exploitants de la Banque sur ses carences ainsi que sur les besoins du marché. 


\section{LE SYSTÈME DE LA BANQUE}

Le système de la Banque de terminologie a été voulu essentiellement simple. Il a été conçu pour répondre aux besoins pressants du milieu canadien en général et tout particulièrement du milieu québécois.

Si nous avons écarté les complexités des structures associatives, des schèmes d'analyse structurale ou d'analyse automatique de textes, ce n'est pas que ces techniques soient sans intérêt, mais bien que nous tenions à faire de la Banque de terminologie, non pas d'abord un instrument de recherche, mais plutôt un outil de consultation axé sur des besoins précis et immédiats.

Nous avons cru de la sorte en faciliter l'accès à tous les usagers, tout en permettant la collaboration la plus large possible à l'échelle des institutions tout autant qu'à celle des personnes. Le succès d'une entreprise comme celle-là tient beaucoup plus à une conjugaison des efforts et des ressources qu'à la complexité de ses structures. Ce n'est ni en dédoublant les efforts, ni en multipliant les banques de terminologie que nous résoudrons les problèmes terminologiques de notre milieu, mais plutôt en canalisant toutes nos ressources vers le même objectif : résoudre d'une façon rationnelle le problème de la recherche, de la coordination et de la diffusion de l'information terminologique.

Robert Dubuc

\section{QUESTION}

M. Provencher : Je voudrais vous soumettre une observation concernant votre critère de pondération. Si j'ai bien compris, le critère est d'abord et avant tout l'Association française de normalisation, et l'Office de la langue française. Je trouve cette solution, si vous me permettez de le dire, un peu ambiguë. L'usage français de l'hexagone n'est pas nécessairement compris hors de l'hexagone.

Réponse : Il me semble qu'il y a deux aspects à votre question. Le premier problème, c'est celui de la normalisation. Nous ne normalisons pas, et si nous acceptons, par exemple, automatiquement, les décisions de l'A.F.N.O.R. c'est parce que ses normes re- posent sur des études sérieuses. Maintenant, quand il s'agit de l'usage québécois, ce n'est pas à nous de légiférer là-dessus. Il y a un organisme officiel au Québec, l'Office de la langue française, qui a tous les pouvoirs légaux nécessaires pour légiférer dans cette matière.

Le deuxième aspect de votre question pose une question de principe : faut-il toujours que la connaissance du terme soit préalable à son emploi ? Ce principe me semble dangereusement restrictif. C'est une question d'acquisition de vocabulaire d'abord et avant tout. Une fois l'acquisition faite il n'y a plus de problème. 